\title{
The High Energy Telescope on EXIST: Hunting High Redshift GRBs and other Exotic Transients
}

\author{
J. Hong', J. Grindlay, B. Allen, \\ Harvard Smithsonian Center for Astrophysics \\ Cambridge, MA, USA \\ E-mail: jaesub@head.cfa.harvard.edu
}

\section{G. K. Skinner,}

NASA/GSFC, Code 661, GSFC

Greenbelt, MD 20771, USA

E-mail: skinner@milkyway.gsfc.nasa.gov

\section{P. Ubertini, L. Natalucci}

Istituto di Astrofisica Spaziale e Fisica Cosmica

Rome, Italy

E-mail: pietro.ubertini@iasf-roma.inaf.it

\section{and EXIST Team}

\begin{abstract}
The High Energy Telescope (HET) on EXIST is designed to locate high redshift Gamma-Ray Bursts (GRBs) and other rare transients fast $(<10 \mathrm{sec})$ and accurately $\left(<20^{\prime \prime}\right)$ in order to allow rapid (<1-2 $\mathrm{min})$ follow-up observations with onboard X-ray/optical/IR imaging and spectroscopy. The HET employs coded-aperture imaging with a $4.5 \mathrm{~m}^{2}$ imaging CZT detector array and hybrid tungsten mask. The wide energy band coverage (5-600 keV) is optimal for capturing these transients and highly obscured AGN. The continuous scan with the wide field of view $\left(90^{\circ} \times 70^{\circ}\right.$ at $10 \%$ coding fraction) increases the chance of capturing rare elusive events such as soft Gamma-ray repeaters and tidal disruption events of stars by dormant supermassive black holes. Sweeping nearly the entire sky every two orbits (3 hour), EXIST will also establish a finely-sampled long-term history of the X-ray variability of many X-ray sources, opening up a new time domain for variability studies. In light of the new EXIST design concept, we review the observing strategy to maximize the science return and report on our recent balloon flight test of a prototype for the CZT detectors needed for HET.
\end{abstract}

The Extreme sky: Sampling the Universe above $10 \mathrm{keV}$ - extremesky2009

Otranto (Lecce) Italy

October 13-17, 2009

\footnotetext{
1 Speaker
} 


\section{Introduction}

The Energetic X-ray Imaging Survey Telescope (EXIST) is newly redesigned to locate high redshift Gamma-Ray Bursts (GRBs) and other exotic transients quickly $(<10 \quad \mathrm{sec})$ and accurately $(<20$ ") in order to allow rapid $(<1-2$ min) followup onboard with optical/IR imaging and spectroscopy [1]. To achieve this, EXIST now consists of a High Energy Telescope (HET), a Soft X-ray Imager (SXI) and an Optical/infrared Telescope (IRT), all mounted on a Spacecraft (in a low inclination, low Earth orbit and with

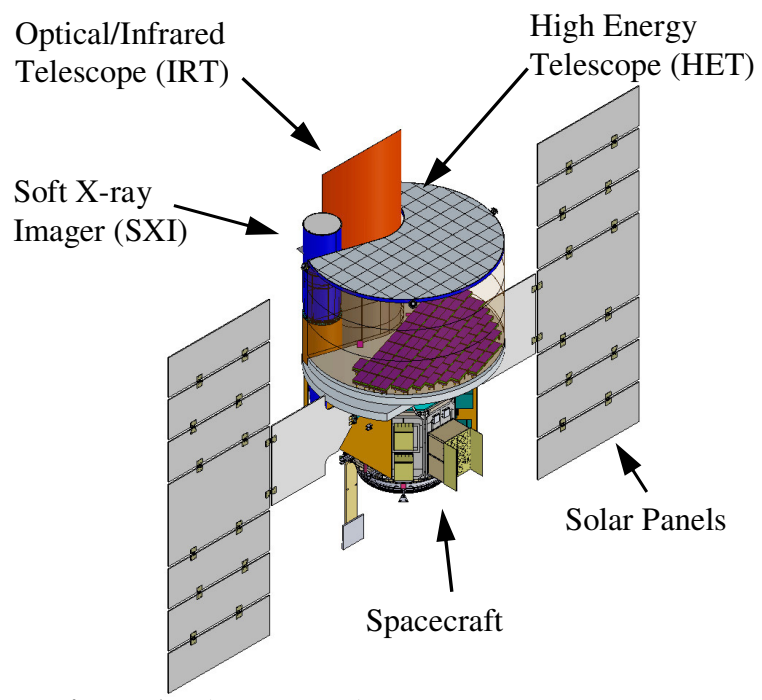

Figure 1: The EXIST observatory rapid slew capability, as shown in Fig. 1. Here we review the overall design of the HET and estimate the broadband sensitivity and sky coverage.

\section{High Energy Telescope}

The hard X-ray band (5-600 keV) is ideal for detecting energetic GRBs, Active Galactic Nuclei (AGN), and transient sources because of the relatively low density of steady background sources and relatively high brightness of transients allowing precise localization. The HET is a wide-field hard X-ray coded-aperture imaging telescope. It will accurately locate GRBs and transients for rapid follow-up with onboard X-ray $(0.1-10 \mathrm{keV})$ and optical-NIR $(0.3-2.3 \mu \mathrm{m})$ imaging and spectroscopy. Redshifts will be derived on board with sensitivity sufficient to measure within (usually) 10 min all those measured (on ground) for Swift GRBs. The HET employs large arrays of fine pixel $(0.6 \mathrm{~mm})$ CZT detectors and a hybrid tungsten mask as shown in Fig. 2a. The key parameters are summarized in Table 1. Like all coded-aperture instruments,

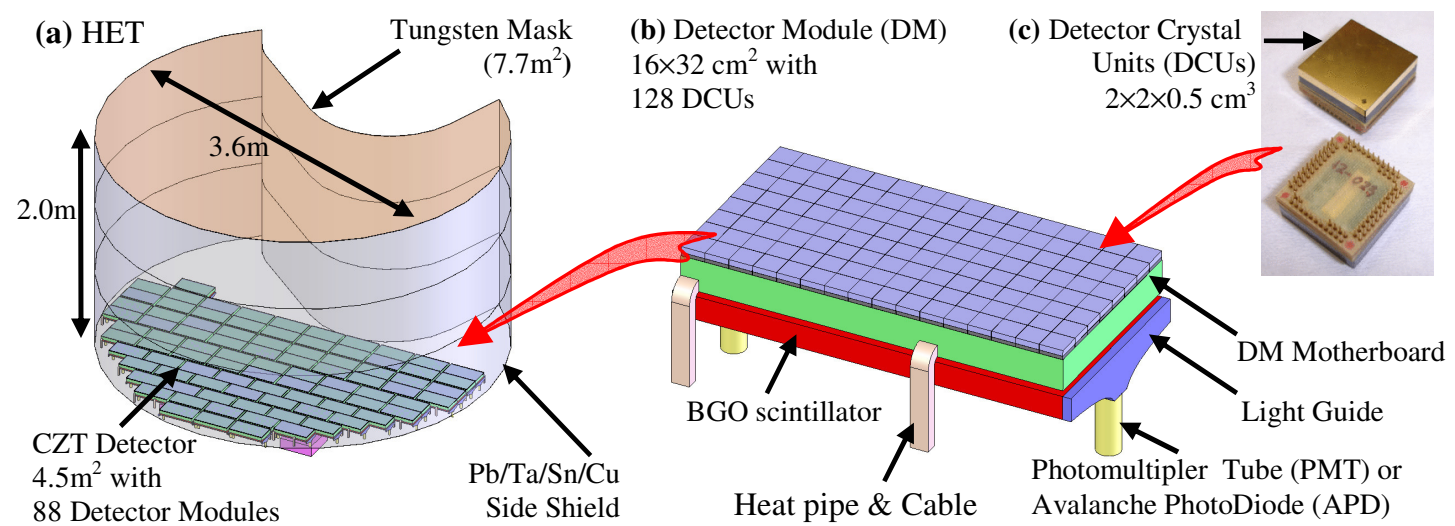

Figure 2: (a) The HET design overview and the CZT detector plane consisting of (b) Detector Modules (DMs), which in turn consist of (c) Detector Crystal Units (DCUs). 
the design is very tolerant to losses of Table 1: The HET parameters. detector pixels or modules. The HET design enjoys a long heritage of successful missions such as Swift and INTEGRAL $[2,3]$.

CZT is the optimal choice for the detectors because of its combination of: (a) high-Z for gamma-ray detection efficiency, (b) good position resolution with the pixilated detectors, (c) low cost, (d) Adequate energy resolution at room temperature, and (e) good Swift experience. The CZT detector plane of the HET is hierarchically modular both in mechanical packaging and in the data concentration and

\begin{tabular}{|c|l|}
\hline Parameters & Values \\
\hline $\begin{array}{c}\text { Telescope } \\
\text { (coded-aperture) }\end{array}$ & $\begin{array}{l}4.5 \mathrm{~m}^{2} \mathrm{CZT}(0.6 \mathrm{~mm} \text { pix, 11.5Mpix }) \\
7.7 \mathrm{~m}^{2} \text { tungsten mask }\end{array}$ \\
\hline Energy Range & $\begin{array}{l}5-600 \mathrm{keV} \text { (imaging CZT) } \\
200-2000 \mathrm{keV} \text { (BGO for GRBs) }\end{array}$ \\
\hline $\begin{array}{c}\text { Sensitivity }(5 \sigma) \\
(\sim 1 \text { y survey) }\end{array}$ & $\begin{array}{l}0.08-0.4 \mathrm{mCrab}(<150 \mathrm{keV}) \\
0.5-1.5 \mathrm{mCrab}(>200 \mathrm{keV})\end{array}$ \\
\hline (10s on-axis) & $\sim 24 \mathrm{mCrab}(<150 \mathrm{keV})$ \\
\hline Field of View & $90^{\circ} \times 70^{\circ}$ (out to $10 \%$ coding) \\
\hline $\begin{array}{c}\text { Angular Res. } \\
\text { Centroiding }\end{array}$ & $\begin{array}{l}2.4^{\prime} \text { resolution } \\
<20^{\prime \prime} \text { for }>5 \sigma \text { source }(90 \% \text { conf. rad.) }\end{array}$ \\
\hline Sky Coverage & Full sky every two orbits \\
\hline Spectral Res. & $\begin{array}{l}3 \mathrm{keV} \\
(3 \% \text { at } 60 \mathrm{keV}, 0.5 \% \text { at } 511 \mathrm{keV})\end{array}$ \\
\hline Time Res. & $10 \mu \mathrm{sec}$ \\
\hline Heritage & $\begin{array}{l}\text { Swift } \mathrm{BAT}, \text { INTEGRAL/IBIS ( and } \\
\text { Fermi/LAT for orbital operation) }\end{array}$ \\
\hline
\end{tabular}
processing. Based on the architecture implemented on Swift/BAT, the CZT detector allows both redundancy and fast imaging but with resolution improved by factors of $\sim 10$ (spatial; angular) and $\sim 3$ (energy).

The detector plane consists of 88 identical detector modules (Fig. 2b), each with 128 Detector Crystal Units (DCUs). Each DCU is made of a $2 \times 2 \times 0.5 \mathrm{~cm}^{3}$ CZT crystal ( $32 \times 32$ pixels, $0.6 \mathrm{~mm}$ pixel) bonded to an EXIST-specific ASIC (EX-ASIC), with a matching 2-D array of $32 \times 32$ channels. The EX-ASIC is essentially the direct bonded (DB)-ASIC developed for the CZT detectors in NUSTAR [4]; but with a modification for lower power and close tiled packaging of DCUs and DMs. The power consumption of the current DB-ASIC is already relatively low $(\sim 80 \mu \mathrm{W} /$ pixel with $\sim 1 \mathrm{keV}$ FWHM resolution and $0.3 \mathrm{keV}$ FWHM electronics noise), but is four times higher than required. Because of the inverse relation between power and noise and the $\sim 2 \mathrm{keV}$ vs. $<\sim 0.5 \mathrm{keV}$ electronics noise (FWHM) requirements for EXIST vs. NUSTAR, the modification for lower power is straightforward. To

(a)

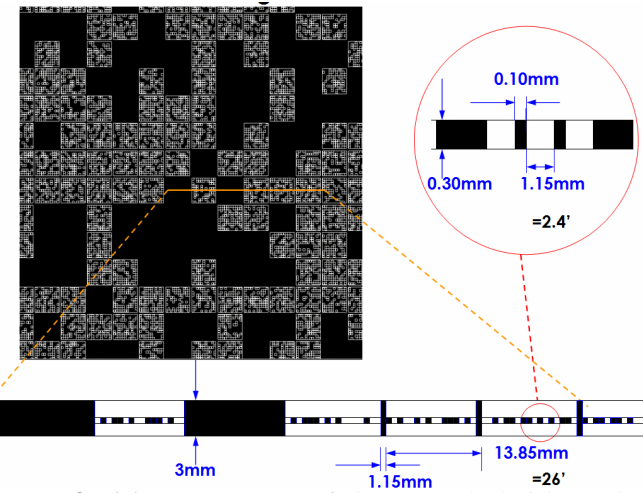

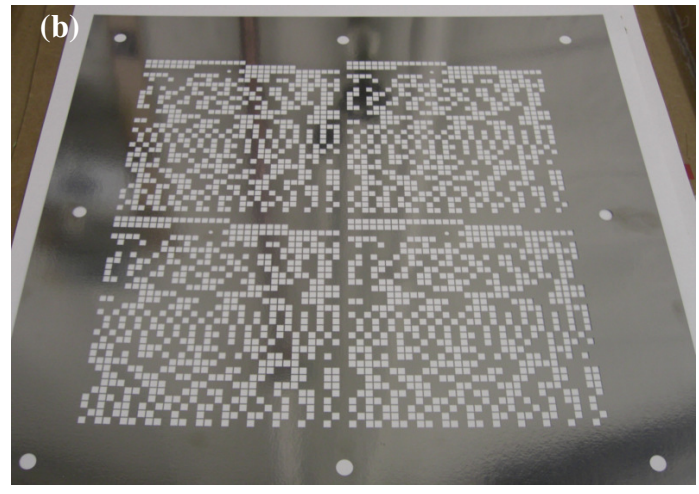

Figure 3: (a) A segment of the HET hybrid mask with cross sectional view: coarse $(15 \mathrm{~mm}$ pitch, $3 \mathrm{~mm}$ thick) and fine $(1.25 \mathrm{~mm}$ pitch, $0.3 \mathrm{~mm}$ thick). (b) A prototype Tungsten mask for ProtoEXIST1 experiment $\left(32 \times 32 \mathrm{~cm}^{2}\right)$. The open holes are chemically etched out from a thin sheet $(0.3 \mathrm{~mm})$. This mask is a $2 \times 2$ URA; full EXIST mask would be random. 
reduce background, the detectors are surrounded by graded- $Z$ passive side shields $(\mathrm{Pb} / \mathrm{Ta} / \mathrm{Sn} / \mathrm{Cu})$ and Bismuth Germanate (BGO) rear anti-coincidence shields as shown in Fig. 2. The latter also extends GRB spectral measurement up to a few MeV.

The hybrid mask, as originally proposed by Skinner and Grindlay (1993) [5], has two scales of pixel elements and thicknesses (Fig. 3), overcoming the difficulty in conventional coded-aperture masks of achieving simultaneously: (1) fine angular resolution, (2) wide energy band and wide field coverage, with minimal auto-collimation, and (3) rapid source localization. The $3 \mathrm{~mm}$ thick coarse elements (15 mm pixel, $50 \%$ open fraction) are effective over the whole 5-600 keV energy band, allowing for the wide field imaging by minimizing offaxis losses due to vignetting (auto-collimation). They also allow rapid FFT calculation of images that can be searched for GRBs and transients. The fine elements $(1.25 \mathrm{~mm}$ pixels, 0.3 $\mathrm{mm}$ thick) are effective below $\sim 100 \mathrm{keV}$. They are superimposed on the coarse mask, giving $25 \%$ overall open fraction below $\sim 100 \mathrm{keV}$ and $\sim 50 \%$ above $\sim 200 \mathrm{keV}$. The fine mask allows the precise location of events found in the rapid initial-onboard analysis. Onboard fineresolution imaging of small regions around candidate locations is then conducted for rapid determination of $<20^{\prime \prime}$ ( $90 \%$ confidence) source locations. Despite the slightly reduced sensitivity of the coarse-mode analysis, the overall detection threshold is not impaired.

\section{Development Roadmap for the HET: ProtoEXIST1, $2 \& 3$}

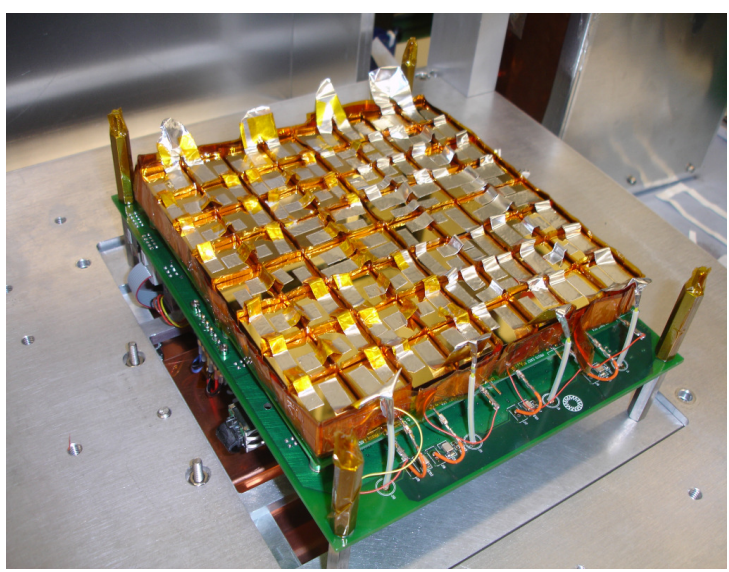

Figure 4: Initial integration of the full $16 \times 16 \mathrm{~cm}^{2}$ CZT detector plane for ProtoEXIST1 on test-stand in lab. HV bias $(-600 \mathrm{~V})$ lines (white) connect to 4 arrays of $2 \times 4$ DCUs on each side.
A series of the balloon-borne experiments are underway to bring the ASIC and electronics packaging for large-array CZT detectors to the point of space qualification for the HET. The first EXIST prototype, ProtoEXIST1, consisting of a $256 \mathrm{~cm}^{2} \mathrm{CZT}$ detector with $2.5 \mathrm{~mm}$ pixels (Fig. 4) and 1024 $\mathrm{cm}^{2}$ Tungsten mask $\left(15^{\circ} \times 15^{\circ}\right.$ at $\sim 50 \%$ coding fraction), had a successful balloon flight from Ft. Sumner, New Mexico, USA on Oct 9, 2009 [6,7]. During the $7.5 \mathrm{hr}$ flight at $39.9 \mathrm{~km}$ altitude, the detector system performed near flawlessly, and a $\quad 65 \mathrm{~min}$ observation of Cygnus X-1 with coarse pointing for all but the final $\sim 8$ min produced a $7 \sigma$ detection of the source as expected, despite a few malfunctions in the pointing and aspect system that resulted in about $6^{\circ}$ offset from the target (and thus $\sim 70 \%$ coding fraction). The detector module in ProtoEXIST1 demonstrates our 
modularization concept to build a very large area CZT detector. The success of the flight is very encouraging for the future development of, next, ProtoEXIST2 with full imaging resolution.

In ProtoEXIST2, we will use the NuSTAR DB-ASIC with its $0.6 \mathrm{~mm}$ pixel resolution CZT but with the tiled DCU modularization concept as in ProtoEXIST1. Then, in ProtoEXIST3, we will employ the EX-ASIC - a lower power version of DB-ASIC. Thus the detector module for ProtoEXIST3 will be identical to that proposed for the HET but will be half the size $\left(256 \mathrm{~cm}^{2}\right.$ for the ProtoEXIST3 vs. $88 \times 512 \mathrm{~cm}^{2}$ for the full HET).

\section{Sensitivity and Sky Coverage}

The 1-year full-sky survey sensitivity of the HET is expected to be $\sim 0.1-0.2$ mCrab, depending on the energy range (Fig. 5). The full mission sensitivity,

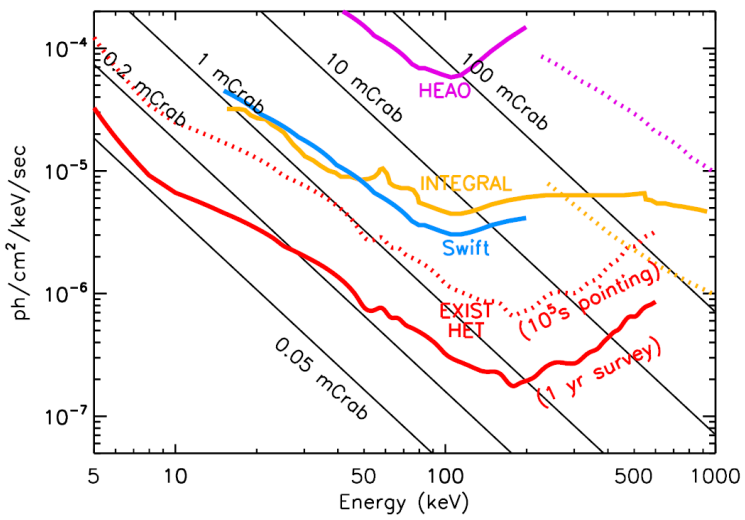

Figure 5: 1-yr survey sensitivity of EXIST/HET and other instruments with $2 \mathrm{y}$ scanning and $3 \mathrm{y}$ sky coverage from pointings, will be $\sim 0.04-0.1 \mathrm{mCrab}$. The continuous scan with the wide field of view $\left(\sim 90^{\circ} \times 70^{\circ}\right.$ at $10 \%$ coding fraction) increases the chance of capturing rare elusive events such as soft Gamma-ray repeaters and tidal disruption events of stars by dormant supermassive black holes. Sweeping nearly the entire sky every two orbits (3 hours, Fig. 6) will also establish a finely-sampled long-term history of the X-ray variability of many X-ray sources, enabling new variability studies of AGN which can constrain SMBH masses. The first $2 \mathrm{y}$ of continuous scanning is also desirable for optimal imaging performance by averaging out ever-present systematic noise. After the initial 2 years of the full sky survey in

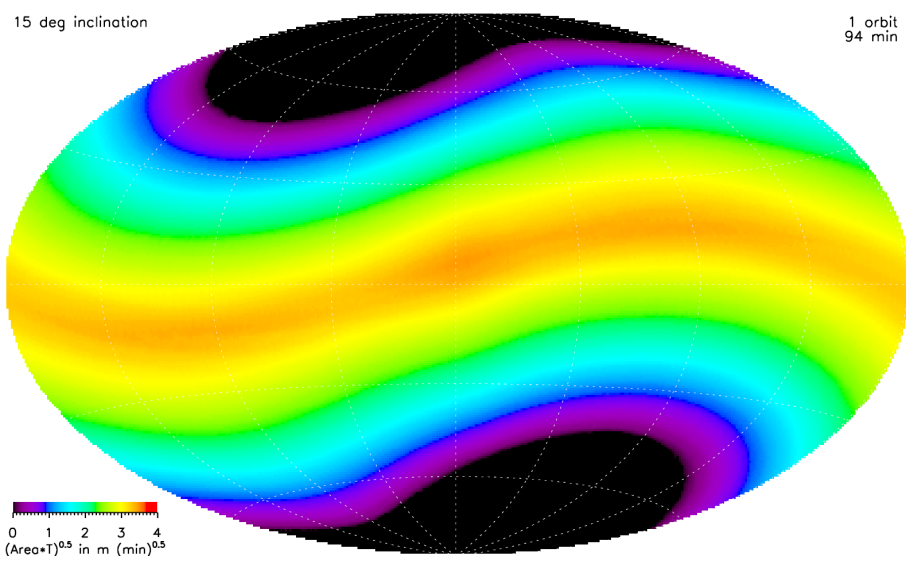

Figure 6: 1-orbit sky coverage of EXIST/HET. The HET will scan nearly full sky every two orbits ( $3 \mathrm{hr}$ ), which is obtained by alternatively scanning above/below the orbital plane by $\pm 25^{\circ}$. scanning mode, EXIST will be primarily in pointing mode for followup studies (X-ray and optical-near-IR spectra, source IDs and redshifts) of selected samples of the $\sim 40,000$ AGNs in the survey. GRB detections and afterglow followups will continue throughout the mission lifetime ( $>5$ years). 


\begin{tabular}{ll}
\hline PSS & PROCEEDINGS \\
\hline
\end{tabular}

\section{Summary}

The HET on EXIST will scan nearly the full sky every two orbits ( $3 \mathrm{hr})$ for capturing GRBs/transients and exploring new variability. Its broadband coverage (5-600 keV) with CZT detectors $(<2-3 \mathrm{keV}$ resolution, FWHM) is ideal for unveiling distant, obscured sources. The HET will localize GRBs with $<20^{\prime \prime}$ accuracy $(5 \sigma)$ and the rapid slew $(<100 \mathrm{sec})$ allows for immediate onboard Optical/IR imaging and spectroscopy of GRB afterglows and prompt redshifts - which enable powerful diagnostics of the early Universe. The advanced CZT imaging detectors required for the HET have been developed through a series of balloon-borne experiments. The $1^{\text {st }}$ generation of the advanced CZT imager with $256 \mathrm{~cm}^{2}$ active area and 2.5 mm pixel (ProtoEXIST1) had a successful flight on 2009 Oct, 9. In the $2^{\text {nd }}$ generation (ProtoEXIST2) we will employ $0.6 \mathrm{~mm}$ pixel CZT imager with the NuSTAR DB-ASICs. In the $3^{\text {rd }}$ generation (ProtoEXIST3) we will use a low power version of the DB-ASIC to meet all the requirements for the CZT imagers in EXIST/HET.

\section{References}

[1] Grindlay, J. et al., AIP Conf.Proc.1133:18-24, 2009 (astro-ph/0904.2210). See http://exist.gsfc.nasa.gov for more information about EXIST.

[2] Gehrels, N. et al., Astrophysical Journal, 611, 1005, 2004

[3] Parmar, A. N. Et al., Proc. SPIE 4851, 1104, 2003

[4] Harrison, F. and NuSTAR Team, BAAS Vol. 41, p.347, 2009

[5] Skinner, G. and Grindlay, J., Astron. \& Astrophys., 276, 673, 1993.

[6] Hong, J. et al., Nuc. Instr. \& Meth. in Phys. Res. Sec. A, Vol 605, 364, 2009.

[7] Hong et al, 2010, in preparation 